\title{
In Focus
Spotlight on the July 23 Issue
}

\author{
Robert A. Gross, MD, PhD, FAAN \\ Editor-in-Chief, Neurology ${ }^{\circledR}$
}

\section{Life-span cognitive activity, neuropathologic burden, and cognitive aging}

Older persons rated late-life and early-life participation in cognitively stimulating activities. After a mean of 5.8 years of annual cognitive function testing, 294 individuals had died and underwent neuropathologic examination. Frequent cognitive activity accounted for an additional $14 \%$ of the variability in rates of cognitive decline, consistent with the cognitive reserve hypothesis.

See p. 314; Editorial, p. 308

\section{Inverse occurrence of cancer and Alzheimer disease:} A population-based incidence study

Cancer risk was reduced by about $50 \%$ in patients with Alzheimer disease (AD), and the risk of $A D$ was reduced by about $35 \%$ in persons with cancer. This observation suggests partially alternative modalities of human senescence.

See p. 322

From editorialists Roe \& Behrens: “...regardless of the particular mechanism underlying the relationship, if future research confirms that the development of cancer and $A D$ are inversely associated, this knowledge may help in gaining a better understanding of and developing new treatments for both diseases."

See p. 310

\section{Differentiating primary progressive aphasias in a brief sample of connected speech}

A brief sample of connected speech was elicited from 62 persons drawn from 3 subgroups of patients with primary progressive aphasia (PPA): nonfluent/agrammatic, logopenic, and semantic. The subgroups were distinguished by easily quantifiable features of speech production. This simple protocol may aid in the differential diagnosis of PPA syndromes.

See p. 329

Syndromes dominated by apraxia of speech show distinct characteristics from agrammatic PPA

This study underscores the importance of proper speech and language characterization in neurodegenerative syndromes. Syndromes dominated by progressive apraxia of speech have different motor speech and neuroimaging characteristics compared to syndromes dominated by agrammatic aphasia. Apraxia of speech should be absent, or at most minimal, to diagnose agrammatic PPA.

See p. 337

\section{Evolution of mild cognitive impairment in Parkinson disease}

Using new consensus criteria, the authors found that one-third of patients with Parkinson disease (PD) already had PD-mild cognitive impairment $(\mathrm{MCl})$ at time of diagnosis, and after 5 years $50 \%$ developed PD-MCl. The new criteria are reliable for clinical practice and may identify patients who will eventually develop dementia.

See p. 346

Reduced postmovement cortical inhibition in patients with paroxysmal kinesigenic dyskinesia
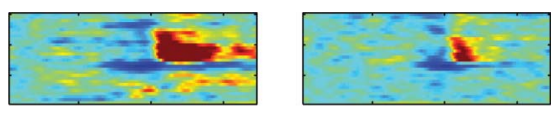

Movement-related oscillation was recorded in 16
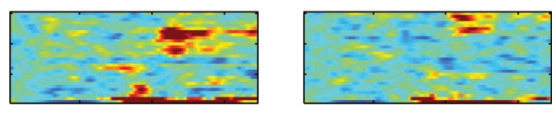
patients with paroxysmal kinesigenic dyskinesia (PKD) and 17 controls, with patients with PKD showing a decreased postmovement inhibition bilaterally. Movement-related electrophysiologic measures may be a marker of the clinical severity of PKD.

See p. 353

GFPT1-myasthenia: Clinical, structural, and electrophysiologic heterogeneity

Some recently emerging neurologic diseases of abnormal protein glycosylation cause congenital myasthenic syndromes. This paper reports on 11 patients with 12 novel mutations in glutamine-fructose-6-phosphate transaminase 1 (GFPT1) harboring hypoplastic endplates and varied defects of neuromuscular transmission. One patient had loss of the musclespecific GFPT1 exon and autophagic myopathy.

See p. 370

Electrographic seizures in pediatric ICU patients: Cohort study of risk factors and mortality

In 550 children in pediatric ICUs, EEG monitoring identified electrographic seizures in 30\%. Electrographic status epilepticus occurred in $38 \%$ and was associated with greater odds of inhospital death after multivariable analysis. Further study is needed to determine whether seizure identification and management could serve as a neuroprotective strategy.

See p. 383

NB: WriteClick is displayed on the Neurology home page. To check out how to submit to this section, point your browser to www.neurology.org and click "Respond" in the WriteClick area. Podcasts can be accessed at www.neurology.org 


\section{Neurology}

Spotlight on the July 23 Issue

Robert A. Gross

Neurology 2013;81;307

DOI 10.1212/WNL.0b013e31829ef315

\section{This information is current as of July 22, 2013}

\section{Updated Information \&} Services

Permissions \& Licensing

\section{Reprints}

including high resolution figures, can be found at: http://n.neurology.org/content/81/4/307.full

Information about reproducing this article in parts (figures,tables) or in its entirety can be found online at:

http://www.neurology.org/about/about_the_journal\#permissions

Information about ordering reprints can be found online:

http://n.neurology.org/subscribers/advertise

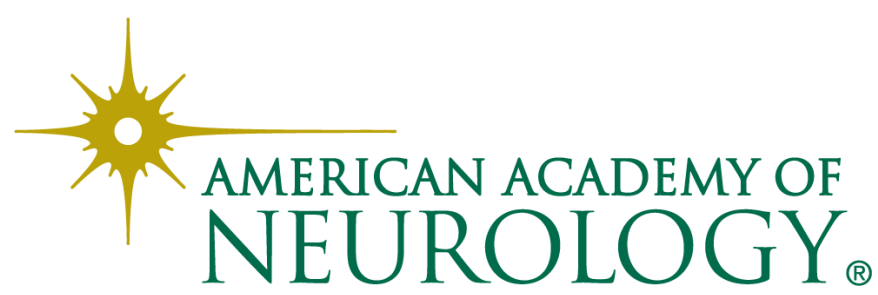

